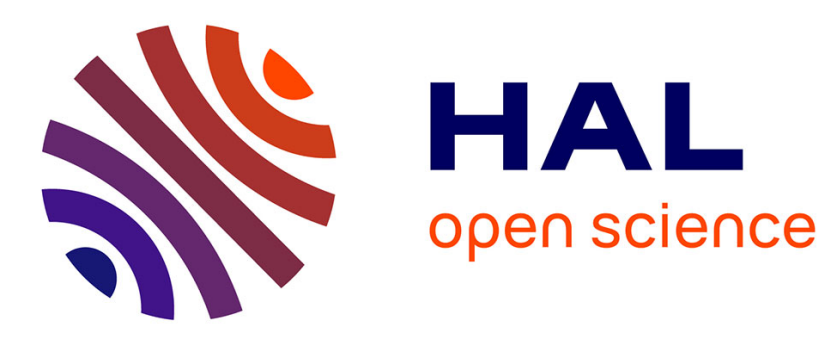

\title{
Anne ou la patience d'être soi selon Kierkegaard
}

Emmanuel Housset

\section{To cite this version:}

Emmanuel Housset. Anne ou la patience d'être soi selon Kierkegaard. Archives de Philosophie, 2013, Sociologie, philosophie: la modernité en question, t. 76 (4), pp.661-684. 10.3917/aphi.764.0661. hal-02142351

\section{HAL Id: hal-02142351 \\ https://hal-normandie-univ.archives-ouvertes.fr/hal-02142351}

Submitted on 28 May 2019

HAL is a multi-disciplinary open access archive for the deposit and dissemination of scientific research documents, whether they are published or not. The documents may come from teaching and research institutions in France or abroad, or from public or private research centers.
L'archive ouverte pluridisciplinaire HAL, est destinée au dépôt et à la diffusion de documents scientifiques de niveau recherche, publiés ou non, émanant des établissements d'enseignement et de recherche français ou étrangers, des laboratoires publics ou privés. 
Emmanuel Housset

Unicaen

Identité et Subjectivité

\section{Anne ou la patience d'être soi selon Kierkegaard ${ }^{1}$}

Comme l'écrit Emmanuel Levinas, l'homme est à la fois verbe et substantif ${ }^{2}$. Il est substantif quand dans l'existence inauthentique et anonyme il est chose parmi les choses, sollicité par les choses matérielles, et en voulant dans l'impatience les posséder toutes, il ressemble à ce qu'il aime. Mais il peut également devenir verbe, se temporaliser dans un accomplissement de soi et ainsi, dans la patience, apprendre à devenir lui-même. La patience n'est pas alors une qualité du sujet, une façon de se posséder soi-même, mais elle est un mode de l'ouverture au monde, un mode de la transcendance, propre à un sujet fini qui fait l'épreuve de sa faiblesse, de sa nudité et de sa pauvreté. La patience est la temporalisation d'un sujet fini dans l'épreuve de l'altérité, que ce soit dans l'amitié, dans la maladie, ou dans la recherche de la vérité. En décrivant ainsi la volonté altérée, brisée, de la patience, il devient possible d'accéder à l'essence de la volonté à partir d'une herméneutique de la finitude.

Avec le christianisme est née cette idée que la patience est la voie du salut, et de ce point de vue il n'y a pas de communauté d'essence entre la patience chrétienne, qui est une grâce, qui est liée à la foi comme rapport entre la personnalité de Dieu et celle du croyant ${ }^{3}$, et la patience païenne, qui n'est qu'un endurcissement en soi, une forme du diabolique comme dira Kierkegaard. En effet, avec le christianisme la patience est comprise comme un devoir d'être soi qui est une temporalisation à partir de l'avenir, et d'un avenir qui est une espérance. Elle demeure une capacité à supporter le monde, les autres hommes et soi-même, mais cette passivité est aussi en elle-même interrogative et agissante. De ce point de vue, la patience ouverte (par rapport à la patience fermée de l'avare) ne consiste pas dans la constitution d'une identité stable au milieu des remous de l'existence, et elle est, au contraire, un renoncement de toute forme d'identité stable pour vivre

\footnotetext{
${ }^{1}$ Ce texte est une version très remaniée d'un article à paraittre en italien dans la revue Tropos.

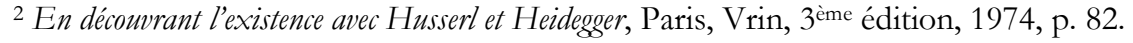

${ }^{3}$ Voir Kierkegaard, Journal, tome 5 1854-1855, Paris, Gallimard, 1961, p. 99.
} 
comme un vrai pauvre de Dieu. Pour ce renoncement à un moi qui se donnerait sa propre ipséité, il faut une longue patience et l'aide de Dieu. Le Christ est la patience, et c'est en cela qu'il est le chemin, la vérité et la vie: «L’homme-Dieu n'a jamais perdu sa patience avec les hommes ${ }^{4}$ ». La patience du père dans la parabole de l'enfant prodigue n'est ni une idée fixe, ni ce qui reposerait sur un calcule raisonné du probable, mais elle se confond avec l'amour, qui est seul capable d'attendre l'improbable. Cette "patience dans l'espérance » (1 Thess 1, 3), selon l'expression de saint Paul, est le fruit même de la charité, qui seule supporte tout. La charité n'est pas une puissance herculéenne face aux attaques du monde, mais elle est une façon toute différente de supporter comme patience combative, enthousiaste et confiante dans la promesse de Dieu. Dans la pensée contemporaine de la subjectivité, Kierkegaard, penseur de la souffrance, a pu à nouveau mettre en lumière en quoi la patience n'est pas une résignation, mais l'humaine réponse à la souffrance à partir de l'amour de Dieu: "Seule cette tension de lutter pour le salut éternel peut vous amener à véritablement tout endurer ${ }^{5} »$. Telle est la thèse difficilement accessible pour notre modernité : penser un devoir d'être soi qui ne se trouve pas lié à la volonté de se donner sa propre ipséité. La question est bien de savoir en retour quelle place il peut rester pour la patience dans une époque de la subjectivité pour laquelle l'ego est la pure présence immédiate à soi, mais aussi la pure possibilité de se réfléchir et de se représenter comme un soi. Kierkegaard semble bien échapper aux critiques sur les métaphysiques de la subjectivité, car, par la considération de la patience, et donc de l'humilité, il montre que la personne ne peut pas répondre à la question « qui suis-je ? » à partir de la seule capacité à se représenter.

La découverte du pouvoir réflexif de la conscience a conduit la pensée depuis Kant à comprendre le sens du monde comme trouvant son origine dans le pouvoir de synthèse de la conscience. Tel serait du moins l'époque du « kantisme » définie après coup par la phénoménologie qui souhaite mettre en lumière un tout autre style de rapport aux choses. Certes, Kant et Husserl ne décrivent pas de la même façon cette synthèse : selon Kant le pouvoir de penser l'objet de l'intuition sensible est l'entendement, et la sensibilité ne contient rien sur la nature des objets. Husserl lui-même va montrer que le sens tient à la fois à la matière et à la visée et qu'il est impossible de dissocier la matière des formes catégoriales ; ainsi la perception est bien un sens qui se constitue dans la sensibilité. Il n'en demeure pas moins que le sens est toujours compris comme une production du pouvoir de synthèse de la subjectivité et de l'intersubjectivité, même s'il s'agit d'une

\footnotetext{
${ }^{4}$ Ibid., p. 98.

${ }^{5}$ Ibid., p. 83.
} 
nouvelle compréhension de la synthèse. Toute la signification de ce que Husserl a nommé «la réduction phénoménologique » est bien de reconduire la réalité au sens et le sens à l'activité de la subjectivité. Dans cette perspective qui libère de toutes les constructions intellectuelles, il s'agit bien de montrer que c'est l'expérience qui prescrit aux choses leur sens de façon à préserver dans cet « empirisme » authentique la richesse et la diversité du réel. Ainsi, le sens est un sens intentionnel relatif au pouvoir de synthèse de la conscience sans lequel il n'y a ni objet, ni monde. Ce qui est alors au moins gagné contre la naïveté, c'est l'idée que le sens n'a rien d'une simple production subjective ou formelle : dire qu'il est quelque chose de l'expérience conduit à dire qu'il n'est ni dans le sujet, ni dans la chose, mais qu'il est ce quelque chose d'identique qui se donne, par exemple, dans la perception. Dans cette purification de la subjectivité, la chose transcendante elle-même n'est que cet identique qui se confirme dans une expérience probante. Dès lors cette conscience pure, comme Husserl le dit dans le $\int 49$ des Idées $I$, est un « système d'être fermé sur soi ${ }^{6}$ », et dans cet idéalisme radical cela signifie qu'il n'y a pas de chose en soi, que toute chose est un sens constitué par la conscience. Il est clair que pour Husserl cela ne revient pas à se fermer à l'altérité des transcendances, puisque c'est au contraire se donner la possibilité de les comprendre en étant attentif à la façon dont elles se constituent dans le vécu. Ainsi le « je » comme origine du sens est la seule vie du sujet, même si Husserl reconnaît l'existence d'une synthèse passive qui participe à cette vie du sujet.

Dans cette compréhension de l'expérience l'étant n'est que l'objectivité de la représentation et se représenter, c'est placer le monde devant soi et toujours d'une certaine façon prédéterminer le visible. La phénoménologie contemporaine a alors tenté de revenir à une expérience plus originaire dans laquelle "l'être sauvage», pour parler avec Merleau-Ponty, se donne comme irreprésentable. Il s'agit alors de retourner à la chose même sans la prédéterminer comme un objet, de rencontrer autrui sans le prédéterminer comme un alter-ego, de recevoir Dieu sans le prédéterminer comme une Idée infinie. Or, cette attention à l'excès même de l'expérience impose de ne plus comprendre la subjectivité comme fermée, mais comme ouverte, et cela remet en cause également sa «perséité » et sa toute puissance. Si l'empirisme naïf avait fait disparaitre le sujet qui considère le monde, l'idéalisme fait lui disparaittre l'altérité en ne la considérant pas comme ce qui excède le pouvoir de synthèse de la conscience et ce qui défait nos représentations. La vie du sens tient également à cette résistance du visible qui est toujours plus que l'image produite par le sujet. La temporalisation de soi ne peut alors être comprise comme la tâche infinie de se vouloir comme être raisonnable, et au-delà de tout projet de purification idéale de la connaissance et de la volonté,

\footnotetext{
${ }^{6}$ Idées directrices pour une phénoménologie, trad. Paul Ricœur, Paris, Gallimard, 1950, p. 163.
} 
elle apparaît plutôt comme une endurance de l'altérité dans la conscience de sa finitude. La remise en cause des philosophies de la volonté pure, par la mise en évidence de l'abîme que toute conscience est pour elle-même, permet de retrouver l'importance de la patience qui avait été considérée jusqu'à la fin de la pensée médiévale comme la racine de toutes les vertus. En effet, la vraie patience ne peut pas être considérée comme la fermeté dans l'auto-détermination de la volonté, tel le voyageur perdu en forêt de Descartes, qui prend la ferme résolution, en l'absence de toute raison de choisir, de conserver toujours la même direction. Elle n'est ni la pure passivité du renoncement, ni la pure affirmation de la résolution, mais elle est cette activité qui répond à la manifestation du monde, qui tente de comprendre ce qui se donne dans le sentir. La patience peut alors être décrite comme une « douceur», qui doit ici être comprise comme une modalité de l'être au monde n'excluant pas une certaine force. Plus exactement, dans cette signification philosophique, la douceur ${ }^{7}$ est cette modalité de la conscience qui ne prédétermine pas, qui ne fixe pas de condition, qui ne force pas le monde à répondre à ses questions. Ainsi la douceur, qui n'est ici en rien doucereuse, est cette souplesse et cette rigueur de la conscience qui tente de recevoir l'altérité, de s'ajuster à elle au lieu de l'ajuster à elle.

Pour pouvoir redonner une place à la patience dans le cadre d'une métaphysique de la subjectivité, pour retrouver la possibilité de voir en elle la forme de toute vie éthique, pour la comprendre comme un respect de la personne qui n'est pas un simple respect pour la loi, il est nécessaire de répondre à plusieurs conditions : envisager une subjectivité finie, une subjectivité dont l'essence est la temporalité, et enfin une passivité qui n'est ni une absence d'esprit, ni une réceptivité, c'est-à-dire une forme de l'activité de l'esprit. Cette passivité qui échappe au découpage habituel des catégories de la pensée moderne, et qui sera si profondément méditée par la phénoménologie française, notamment Emmanuel Levinas et Henri Maldiney, est de l'ordre de la rencontre et ne doit pas être pensée dans le cadre de la constitution d'un objet par un sujet. La patience peut être retrouvée comme le lieu du sens, le lieu dans lequel je peux comprendre quel est mon devoir, parce qu'elle n'est pas un enfermement dans l'immanence, mais une exposition au monde. Elle ne peut donc se concevoir comme une forme a priori de la vie éthique qui ferait du devoir une production de la conscience, et elle est plutôt cette attente qui laisse la conscience de ce que j'ai à faire naître de la rencontre elle-même. Retrouver la patience comme vertu fondamental du philosopher et donc de l'existence est ce à quoi invite Kierkegaard dans cette pensée du moi qui ne peut renaître à lui-même qu'à partir de la conscience de son imperfection. Si on entend par «moi » le pur pouvoir de dire « je », sans le confondre avec le «soi » produit de la réflexion" ${ }^{8}$, ce

\footnotetext{
7 Voir notre article, «La douceur de la patience », Revue d'éthique et de théologie morale, n²50, 2008.

8 Voir les analyses historiques de Vincent Carraud, L'Invention du moi, Paris, PUF, 2010.
} 
pouvoir de dire «je » a lieu dans la patience, c'est-à-dire devant ce qu'il rencontre. Ainsi, en retrouvant l'humilité, en mettant en cause le sujet moderne fondé sur le projet d'une totale main mise sur soi, Kierkegaard invite à comprendre une passibilité propre à la persévérance dans laquelle l'autre homme me déborde. L'absence de patience est une insensibilité spirituelle et avec la reconnaissance de la patience a lieu un autre rapport à la vérité dans lequel elle n'est pas ce que je produis et dont je serais propriétaire, mais ce qui m'affecte et m'éclaire. En outre, dans tout ce renversement conceptuel, cette patience du sujet fini qui se temporalise en sachant qu'il ne peut se donner seul sa propre ipséité, est animée par une inquiétude spirituelle suprême, par la crainte et le tremblement, par une « impatience de l'éternité ${ }^{9} »$.

Kierkegaard dans les Dix-huit discours édifiants ${ }^{10}$ décrit cette patience attentive, qui n'est ni purement passive, ni purement active, en commentant la parole de l'Evangile «C'est dans la persévérance (bypomonê) que vous posséderez vos âmes»(Luc, 21, 19). En comprenant la persévérance comme le lieu du salut, Kierkegaard peut donner à penser une patience qui échappe à l'alternative de la pure volonté et de la pure passivité, et c'est pourquoi il va pouvoir comprendre cette patience comme la vertu du temps. Dans cette perspective, la persévérance n’est pas une qualité extérieure que l'homme pourrait avoir ou ne pas avoir, mais elle est la perfection de la possession de soi, de l'accomplissement de soi dans la finitude de l'action. Or, pour élucider la patience comme la temporalité du soi authentique, Kierkegaard commence par montrer en quoi elle est liée à la nudité de l'homme : «Un homme vient nu au monde ; il n’apporte rien avec lui »", mais il ne s'agit pas de répondre à cette nudité par la main vide qui peut tout devenir, comme Aristote a pu la donner à penser. Certes, l'homme doit apprendre à se posséder lui-même, parce qu'il ne possède rien, mais justement « la vie doit être conquise et possédée dans la persévérance » ${ }^{12}$, et il s'agit là du nom même du courage, de la force d'âme sans laquelle on ne peut pas devenir soimême, ni même accéder au devoir de devenir soi. La vie n'est ni pure intuition, ni pure réflexion, mais elle est la persévérance elle-même qui n'est pas liée au maintien d'une identité stable, car elle est l'acte de risquer sa vie. Kierkegaard laisse ainsi l'bypomoné de la Bible des Septante travailler au cœur de la métaphysique de la subjectivité, afin de réactualiser cette idée d'une constance dans le désir qui surmonte l'épreuve de la durée, et qui est en cela une attente obstinée et combattante. Il utilise alors la métaphore du voyage : l'homme patient est tel à ce voyageur qui évite une trop grande hâte, qui sait se reposer, car il sait que ce sont les conditions pour aller loin. L'existant est

\footnotetext{
${ }^{9}$ Journal, op. cit. p. 77.

10 Ils seront cités d'après le tome 6 de la traduction de Paul-Henri Tisseau et Else-Marie Jacquet-Tisseau des CEuvres complètes, Paris, Editions de l'Orante, 1979.

11 OC, 6, p. 148.

12 Ibid.
} 
in via et il doit prendre son temps, c'est-à-dire ni se forcer, ni forcer le monde. Il est clair que l'impatience peut devenir une errance dans ce chemin qu'est l'existence, parce qu'on ne peut alors se proposer que des buts proches. Ainsi, dans le devenir, l'homme n'est pas d'abord lui-même, et le plus difficile n'est pas d'attendre les choses extérieures, mais de s'attendre soi-même. Or bien évidemment on ne s'attend pas soi-même comme on attend les biens extérieurs et l'âme est une contradiction en ce qu'elle ne peut être ni acquise, ni perdue. En conséquence, «si l'âme est bien cette contradiction on ne peut la posséder qu'en l'acquérant et on ne peut l'acquérir qu'en la possédant $»^{13}$. Cela signifie que l'homme n'est lui-même que dans le mouvement où il se cherche, et l'âme ne doit pas être comprise comme un substantif, mais comme l'acte même de se porter vers soi. Tel est le paradoxe : l'âme est inamissible et pourtant on ne la possède qu'à la devenir. Ainsi tout ce thème de la nudité montre que l'éloge de la persévérance ne consiste pas à en rester à une conception abstraite du moi, selon laquelle il procéderait par pure identité et serait son propre maître. En effet, dire que l'âme n'est possédée que dans l'acte même de l'acquérir écarte l'idée simple d'une autoposition, d'une auto-affirmation du moi. Devenir soi, cela consiste à s'accomplir comme un être spirituel qui s'ouvre à l'éternel dans le temporel. Comme Kierkegaard le met en lumière dans La maladie à la mort, l'impatient qui veut être César ou rien désespère nécessairement de lui-même. Or cette maladie de l'esprit qu'est l'impatience n'a rien d'occasionnelle ou d'accidentelle, et elle est au contraire l'état le plus habituel de l'esprit. L'analyse réflexive montre bien qu'être un esprit, c'est structurellement être en crise, c'est ne pas être soi, et dans cette perspective la crise est la vie même de l'esprit. Dès lors, il convient de reconnaître que c'est toujours à partir de l'impatience que l'on parle de la patience et qu'il n'est possible de penser la patience qu'à partir de la tâche d'être soi, de devenir soi et de se réconcilier avec soi. La patience suppose donc un choix absolu, une résolution, qui pose l'éthique en se rapportant à la fois au temporel et à l'éternel ${ }^{14}$.

Il est alors possible de distinguer trois formes de cette maladie de l'âme, de ce désespoir, qu'est l'impatience. Il y a d'abord l'impatience de celui qui ne sait pas qu'il veut un bien éternel et qui papillonne de bien matériel en bien matériel, dans une sorte de zapping spirituel devenu une véritable addiction. Néanmoins, il y a une autre forme de l’impatience, celle de la faiblesse de l'âme dans laquelle on n'a pas le courage d'être soi. Dans cette faiblesse constitutive de la volonté, l’individu se trouve totalement dépossédé de lui-même. Enfin, la troisième forme de l'impatience négative est celle qui est liée à la volonté effective d'être soi, mais dans le constat de la difficulté à

\footnotetext{
13 OC, 6, p. 151.

${ }^{14}$ Voir L'alternative, OC, 4, p. 162. Voir aussi de Jacques Colette, «Le don et la tâche », sans Soren Kierkegaard. Pensée et problèmes de l'éthique, Anne-Christine Habbard et Jacques Message éds, Presses Universitaires du Septentrion, 2009.
} 
l'être. De façon évidente, avec ces trois modalités l'impatience n'a rien d'un état psychologique contingent, mais elle prend une signification universelle, dans la mesure où elle est le mode d'être de l'esprit. L'homme vit dans l'impatience tant qu'il est dans la non-vérité par rapport à lui-même, et il doit prendre conscience de cette impatience pour devenir véritablement esprit. Ainsi la persévérance est cet effort qui consiste à apprendre à vouloir être soi, ce qui est évidemment la tâche la plus difficile. En effet, selon la première forme de l'impatience les hommes veulent ce «bonheur terrifiant ${ }^{15}$ » qui consiste à posséder le monde. Si la philosophie est une folie pour l'homme qui appartient au monde, vouloir conquérir le monde est une folie pour le philosophe, car cela enferme l'homme dans les biens matériels et le ferme à cette promesse qu'il est lui-même.

Dans cette lecture de l'Evangile de Luc, Kierkegaard insiste sur une précision qui lui semble décisive : on devient soi non pas « grâce à » ou « par » la persévérance, mais « dans » la persévérance, car celle-ci n'est pas un moyen parmi d'autres, mais le «style ${ }^{16} »$ même de la vie de l'homme qui se veut vraiment lui-même. En effet, la persévérance n'est pas un bien comme les autres et elle n'est pas un outil qu'il est possible de prendre puis de déposer : l'homme se possède dans la patience et dès qu'il renonce à la patience il se perd lui-même. Ainsi, non seulement la patience n'est pas un bien du monde, mais elle n'est pas non plus ce pouvoir de mise à disposition du monde et de soimême. En outre, dans la volonté de posséder le monde, l'impatience est parfois bien plus utile, bien plus efficace, et d'une certaine façon le monde appartient aux impatients, à ceux qui, tel Alexandre tranchant le nœud gordien, veulent tout immédiatement. Dès lors, toute patience forcée n'est qu'une forme d'impatience, une béquille face à l'impossibilité extérieure de céder à la précipitation ; ainsi l'avare sait attendre de façon remarquable, mais son attente n'est qu'une forme sophistiquée de sa volonté de tout posséder le plus vite possible. Pour le formuler d'une manière paradoxale : sa patience n'est qu'un instrument au service de son impatience. La véritable patience ne vient pas d'une condition extérieure, c'est-à-dire de l'impossibilité matérielle de tout obtenir tout de suite, et en cela elle n'est pas une ruse de l'impatience. Kierkegaard citant encore l'Evangile de Luc $(8,15)$ «Le bon grain ne vient à maturité que dans la patience », montre que la patience n'est pas un simple moyen, mais la maturation même du moi, et c'est pourquoi la patience engendre la patience. En outre le moi lui-même n’est pas le produit d'une réflexion, ni la capacité d'agir selon

\footnotetext{
15 OC, 6, p. 153: «Le bonheur suprême que les hommes convoitent en leur folle impatience sans savoir ce qu'ils veulent, bonheur terrifiant pour qui les a vus y parvenir, et qui consiste à s'assurer la possession du monde et à s'en être assuré, c'est là le premier objectif de l'homme, pourtant si loin d'être son but qu'il faut bien plutôt s'en éloigner ». ${ }^{16}$ En donnant au terme de style le sens fort et authentique d'un mode du rapport à la vérité et du rapport à soi. Ce style est justement ce que je ne peux pas produire, mais qui résulte de la patience de ma réponse. Voir Postscriptum définitif et non scientifique aux miettes philosophiques, tome 10, p. $81:$ : Il en est ici comme d'avoir du style : seul en a vraiment celui qui n'a jamais rien de tout prêt, mais qui, chaque fois qu'il se met au travail "agite les eaux du langage" (cf. Jn 5, 4) de sorte que le terme le plus courant prend vie à ses yeux comme s’il était tout neuf ».
} 
les représentations des lois. Si la volonté est la forme du moi, comme pour Fichte, vouloir, c'est consentir à une vérité qui s'annonce. Le moi n’est pas ce qui se produit lui-même, mais ce qui en soi dit « oui » au don de la liberté. Le seul « je » authentique serait alors cet acte de parole du « oui » et du «merci $»^{17}$. Néanmoins, la patience ne peut être ainsi comprise comme le chemin du salut qu'à partir de l'évidence première qu'on ne se possède pas soi-même, que l'homme d'abord n'est pas lui-même et qu'il ne s'agit pas simplement d'un manque d'éveil à soi comme dans les philosophies de la volonté pure. Contrairement à ce que l'homme imagine de lui-même, il ne se veut pas d'abord lui-même et le chemin de la patience n'est autre que la possibilité d'apprendre à se vouloir. Il y a là un paradoxe déjà longuement médité par saint Augustin, « se vouloir » semble être une chose si naturelle que cela ne peut pas faire l'objet d'un impératif, et pourtant cela suppose une conversion, qui est peut-être la tâche la plus difficile. En effet, ce renversement de tout son être tient dans « la patience de comprendre que pour acquérir son âme dans la patience, il faut faire acte de persévérance $»^{18}$. Ainsi on ne gagne la patience que contre la passion de l'impatience, contre ce pathos de l'immédiateté, qui est d'une essence tout à fait différente de la passion positive de la foi, du pathos éthique et religieux. En conséquence, la patience n'est pas une pure passivité et " "Acquérir son âme" incline d'emblée l'esprit vers une tranquille mais inlassable activité »". Ici le calme n'est pas la passivité, bien au contraire :

« Il n'est pas question de prendre d'assaut, de pourchasser, de chercher à saisir quelque que chose, mais de demeurer dans un calme toujours plus grand ; car ce qui doit être acquis est au dedans de nous et le malheur vient de ce que nous sommes à l'extérieur de nous-mêmes ; car ce qui doit être acquis est dans la patience, caché en elle, non point de telle sorte qu'en effeuillant pour ainsi dire patiemment la persévérance l'on finirait par trouver son âme qu'au milieu d'elle, mais c'est au cœur même de la patience que l'âme s'enferme, tissant patiemment sa toile pour prendre possession de la patience et d'elle-même $»^{20}$.

La patience n'est donc pas une technique, un procédé qui permettrait de se trouver, et d'une façon générale il n'y a pas de «technique du soi». Dire que la patience est le chemin, c'est reconnaître en elle l'essence même de l'âme : la patience est un pouvoir de rassemblement d'où provient l'âme. Dans cette opposition du calme et de la précipitation comme deux styles d'existence, comme deux modes de l'activité, Kierkegaard veut mettre en évidence qu'acquérir son

\footnotetext{
${ }^{17}$ Voir Jean-Louis Chrétien, La voie nue, Paris, Minuit, 1990, chapitre 8 « Le moi et le péché selon Kierkegaard », p. 171

18 OC, 6, p. 156.

${ }^{19}$ OC, 6, p. 157.

${ }^{20} \mathrm{Ibid}$.
} 
âme, ce n'est pas comme s'approprier un bien matériel, et cela d'abord parce qu'il est nécessaire de se libérer d'une possession apparente de soi : la patience est d'abord un exode par rapport à un moi illusoire. Cela permet à Kierkegaard d'inverser l'argument de la force : la patience est l'arme du faible, car elle n'est pas une possession, mais une dépossession. Il ne s'agit pas de redoubler de vigueur, de forcer le monde à répondre à ses questions pour qu'il réponde à ses besoins, mais de renoncer à une possession tout à fait illusoire de soi. Toute cette analyse met donc en lumière qu'il y a un nécessaire abandon dans la patience, qui n’est ni passivité, ni lâcheté, mais humilité :

«Dans la patience l'âme transige avec tous ses possesseurs, avec la vie universelle, se rachetant d'elle par la souffrance, avec Dieu en se recevant de lui par la souffrance, avec elle-même en gardant ce qu'elle donne elle-même aux deux sans que personne ne puisse lui ôter la patience $»^{21}$.

L'âme ne peut rien obtenir par la force et le devenir soi suppose toujours un renoncement au projet de possession du monde, or c'est justement dans cette faiblesse qu'elle sera plus forte que le monde. En effet, la tâche d'être soi, c'est-à-dire le devoir de devenir soi et de se réconcilier avec soi, se dévoile comme un apprentissage de la patience, car le fond de la subjectivité ne peut pas être le moi rivé à soi. Pour reprendre la célèbre thèse de La maladie à la mort, « le moi est un rapport qui se rapporte à lui-même », ce qui signifie que le devenir de l'esprit est en premier lieu la réflexion sur soi du rapport de l'infini et du fini, de l'éternel et du temporel. Le moi tel qu'il est mis ici en évidence n'est pas le rapport, mais cette possibilité que le rapport se rapporte à lui-même. On ne devient soi que dans la patience, précisément parce que le moi n'est pas un état, mais un devenir et un devoir-être. Si les philosophies de la volonté pure ne peuvent laisser aucune place à la patience, parce qu'elles s'en tiennent au pouvoir d'auto-appropriation de la subjectivité, Kierkegaard, en partant de la nudité et de la pauvreté de l'homme, peut retrouver la patience comme le chemin même d'une subjectivité exposée. Ainsi pour lui la patience est la synthèse du temporel et de l'éternel : l'homme est pris entre le temporel qu'il ne peut fuir et l'éternel qu'il doit vouloir. A la fois l'homme doit endurer le temporel et se porter calmement vers l'éternel ; il y a donc bien une synthèse des deux dimensions de la patience, notamment dans la plénitude de l'instant, ou plus précisément la patience est elle-même la synthèse de l'endurance et de l'espérance. De cette façon, dans cette synthèse qu'est l'existence patiente, la patience permet de ne pas être écrasé par la nécessité et de ne pas s'obstiner non plus dans des possibles imaginaires, et c'est pourquoi cette vertu biblique est la vertu du temps : tenir ensemble le possible et le nécessaire dans le devenir. Il est alors envisageable de dire que le moi est la réflexion sur ce rapport de l'éternel et du temporel,

${ }^{21}$ OC, 6, p. 158. 
du possible et du nécessaire. En conséquence, pour Kierkegaard, l'homme devient un moi, un esprit, en se déterminant selon une fin qui ne se trouve ni en lui, ni dans le monde fini. Telle est la vraie patience : elle consiste à appartenir à la vérité dont on fait l'épreuve, et en ce sens la patience est ce qui fait comprendre qu'on ne se possède pas soi-même et que devenir soi ne revient pas à vivre dans l'obsession d'une fin et d'une identité que l'on se donne à soi-même ${ }^{22}$. Néanmoins cet éloignement de soi vers les fins éternelles doit permettre de s'engager dans la temporalité, et telle est la patience, à savoir une existence toute en tension entre l'éternel qui demeure et le temporel qu'il faut assumer ${ }^{23}$. Kierkegaard décrit donc ici l'essence de la temporalité éthique qui est la synthèse de la patience dans le monde et de l'impatience de l'éternité.

Bien sûr, il y a une patience de la connaissance de soi, mais cette patience ne peut pas être la vraie patience quand elle n'est qu'un moyen de la connaissance, qui se trouve lui aussi déposé une fois la connaissance acquise. En respectant la lettre du texte de saint Luc, Kierkegaard montre que la patience véritable revient à exister dans la patience en agissant également en fonction de la vérité reçue précisément dans la patience. Il s'agit d'exister cette vérité : «Quiconque veut acquérir son âme dans la patience reconnaît qu'elle ne lui appartient pas et qu'il y a une puissance d'où il lui faut la retirer, dont il reçoit cette acquisition qu'il doit faire lui-même» ${ }^{24}$. Dès lors, ici la patience ne relève pas d'un rapport direct à soi, elle n'est pas une autodétermination pure indépendante de son objet et elle ne suppose pas pour autant de conditions extérieures. En échappant à l'alternative de l'autonomie et de l'hétéronomie propre aux philosophies réflexives, la métaphysique de la subjectivité de Kierkegaard souligne que l'homme ne devient conscient de lui-même que devant ce qui le fonde et il ne s'agit pas seulement du pouvoir de se représenter comme un soi, mais bien également du pouvoir même de dire «je». Si le moi était autofondateur et autotélique, l'unique tension serait interne: soit le moi se veut lui-même, soit le moi ne se veut pas lui-même. L'impatience ne serait alors que l'incapacité à tenir la distance qui va de soi à soi et elle se réduirait à une faiblesse de la volonté par rapport à l'idéal herculéen d'un au-delà de toute finitude. Kierkegaard veut au contraire décrire qu'il n'y a pas de repos en soi et que vivre en fonction de l'idéal imaginaire d'une totale indépendance est ce qui provoque une crise insurmontable au lieu de la résoudre. Chercher le repos en soi, c'est le chercher où il n'est pas ; mais il n'est pas non plus dans le monde. Dans cette perspective, la patience authentique n'est pas celle d’Ulysse, qui, en dépit

\footnotetext{
${ }^{22}$ Voir Jacques Colette, Kierkegaard et la non-philosophie, Paris, Gallimard, coll. TEL, 1994, p. 128 et également p. 129 : «La patience, sans laquelle ne peut se concevoir le style de la communication indirecte, s'impose pour prévenir l'impatience absolue de la spéculation, laquelle, d'ailleurs, n'est pas incompatible avec le long travail du concept. Elle diffère donc de la patience du négatif $»$.

${ }_{23}$ Sur ce rapport de l'éternel et du temporel voir Jacques Message, «La possibilité infinie, instance transcendantale de l'éthique », dans Søren Kierkegaard. Pensées et problèmes de l'éthique, Presses Universitaires du Septentrion, 2009.

24 OC, 6, p. 160.
} 
de tous les obstacles, veut revenir dans sa patrie, mais elle est celle de Moïse qui naît en une terre étrangère et qui se trouve conduit ailleurs vers une terre promise. Ainsi la patience ne doit pas être une forme du démoniaque ${ }^{25}$ qui consiste à s'enfermer en soi, mais elle doit se comprendre comme une patience qui revient à la puissance qui pose l'homme. Le seul vrai repos, celui qui est compatible avec la finitude de notre existence, est celui qui est un mouvement vers l'origine qui nous pose.

Dans cette perspective, l'autonomie est une connaissance impatiente comme non-respect du temps, car elle est une pure affirmation de soi qui ne peut être que l'expérience de notre impuissance à nous tenir nous-mêmes et à nous vouloir nous-mêmes, ce qui engendre le désespoir. En outre, la purification toujours plus poussée de la patience dans les philosophies de la volonté, de façon à lui retirer toute forme de passivité, ne conduit qu'à un moi toujours plus abstrait, à la liberté vide d'un moi autocréateur et qu'à une pure volonté de volonté du moi qui se veut lui-même. Or cette absolutisation de la volonté pure est finalement la véritable maladie mortelle. Notamment selon la représentation stoïcienne de la patience, le moi veut être son propre maitre, mais il s'agit d'un roi sans royaume réduit à l'arbitraire de sa propre volonté26 ${ }^{26}$ Il n'y a donc de vraie patience que dans la conscience de ce qui nous pose, qu'en surmontant l'ignorance de notre impuissance à nous poser nous-mêmes. Le moi théologique est précisément celui qui découvre que Dieu est ce qui donne un avenir et qui est source de toute patience, parce qu'il fait prendre conscience de sa nonvérité. La patience proprement humaine n'est plus alors comparable à celle d'Ulysse qui veut retrouver son chez soi, mais elle se comprend à partir de la patience d'Abraham qui se reçoit de la parole de Dieu et qui s'approche d'une vérité qu'il n'est pas et qu'il ne peut pas posséder. Dans Crainte et tremblement Kierkegaard analyse la patience d'Abraham, c'est-à-dire la foi en la promesse de Dieu, en la distinguant de la résignation, qui est pourtant aussi nécessaire au salut, mais qui n’est pas encore une sortie hors de soi, hors de l'immanence, même si elle est déjà une forme de renonciation à soi. Abraham n'est pas un exilé qui pleure sur ce qu'il a perdu, car pour lui il est plus grand de croire en gardant la promesse ${ }^{27}$.

Il faut reconnaître que la force des analyses de Kierkegaard dans les Dix-buit discours édifiants est de partir de cette idée que la patience de la réflexion n'est pas ce qui s'impose d'emblée par son utilité et qu'elle est finalement de peu de poids par rapport au charme de l'impatience qui flatte le désir. Dès lors, si la patience est douce, elle n'est pas, elle, doucereuse, et elle est cette force qui

\footnotetext{
${ }^{25}$ Voir Jean-Louis Chrétien, Le regard de l'amour, Paris, Desclée de Brouwer, 2000, chapitre V « Perdre la parole. Le démoniaque selon Kierkegaard ».

26 Bien évidemment il serait possible de discuter cet usage du terme « moi » et du concept de «volonté » dans le stoïcisme, néanmoins il s'agit avant tout pour Kierkegaard d'opposer deux essences inverses de la patience, c'est-à-dire de deux manières d'être dans la patience qui sont totalement opposées.

27 Voir OC, 5, p. 112-113.
} 
accueille tout être comme une promesse ; elle est alors également cette ouverture de la sensibilité qui brise le cercle de l'immanence. La patience est ainsi une humilité qui dépasse le simple projet d'être soi dans la fidélité à soi qui va au-delà de soi pour accomplir le devoir d'être soi. En cela, « la patience n'apprend pas à se méfier de la vie $»^{28}$, car elle est cette confiance en la vie qui vient de Dieu. Ainsi, Kierkegaard met en évidence cette vérité eidétique qu’il est impossible de penser la patience sans la confiance en la vie elle-même, confiance qui n'a rien à voir avec la certitude de son propre pouvoir. Or cette confiance contient en elle la possibilité même de l'endurance de l'altérité :

« Garder son âme dans la persévérance, c'est-à-dire la maintenir enchaînée dans la patience afin qu'elle n'en sorte pas et se perde quand commence la longue lutte contre un ennemi qui ne se lasse jamais, le temps, et un autre, aux multiples aspects, le monde $»^{29}$.

La patience est alors cette capacité de renoncer à tout ce qui appartient au temps et au monde et elle n'est pas l'obstination à maintenir le souvenir du passé, elle n'est pas l'idolâtrie du monde, autrement dit elle n'est pas la volonté désespérée de s'accrocher à ce qui passe dans la nostalgie de ce qui est perdu. Dès lors on ne surmonte pas le temps en cherchant à abolir le temps, par la vaine tentative de lui retirer sa dimension de perte, mais en ayant vraiment la vie devant soi dans la confiance en la promesse. La seule véritable alternative de l'existence est alors celle de la dispersion vers les biens du monde ou de la tension vers l'avenir de Dieu, et c'est pourquoi l'endurance seule, coupée de la confiance, n'est qu'une vaine tentative pour surmonter la dispersion qui ne libère pas de la dispersion et qui demeure finalement une forme de l'impatience :

«L'impatience peut ainsi revêtir des formes nombreuses. Au début, on la reconnaît à peine, tant elle est douce, conciliante, exaltante, mélancolique, pleine d'attrait et de compassion ; mais ayant épuisé tous ses artifices, elle finit par hausser le ton, vous tenir tête et vouloir tout expliquer bien qu'elle n'ait jamais rien compris $»^{30}$.

La véritable patience ne vise pas à revêtir le monde de ses désirs et de ses projets, mais elle est une intelligence, une capacité à comprendre les situations ; elle est cette sagesse pratique qui sait agir à partir d'une attention au prochain, et c'est pourquoi Kierkegaard identifie la patience et la pitié : «Elle est en vérité le bon Samaritain dont parle l'Ecriture $»^{31}$. Une telle identité d'essence entre pitié et patience est tout à fait décisive, car elle est cette proximité dans la sensibilité qui sait

\footnotetext{
28 OC, 6, p. 177.

${ }^{29} \mathrm{Ibid}$.

30 OC, 6, p. 180.

31 OC, 6, p. 181.
} 
agir. En elle action et contemplation ne se séparent pas : elle agit en comprenant et elle comprend en agissant. Le bon Samaritain n'est ni celui qui se détourne, ni celui qui se lamente devant la richesse du monde, mais celui qui comprend qu'il est encore temps pour faire quelque chose, non pas demain, mais dans l'aujourd'hui de Dieu, dans un présent lourd de l'attente de Dieu. L'homme patient n'est pas alors celui qui compte le temps passé à attendre devant l'impossibilité de tuer le temps et il est au contraire celui qui est riche de son avenir, parce qu'il vit dans l'attente de l'éternel en entretenant l'espérance. Une telle plénitude du temps dans l'attente n'est pas pour Kierkegaard la simple anticipation du vraisemblable ${ }^{32}$, et c'est pourquoi elle est l'offrande même de l'âme. Dans cette véritable phénoménologie de l'espérance, Kierkegaard met en lumière que la patience ne se trouve ni dans la dolence de l'habitude, ni dans l'imagination d'un avenir vraisemblable, et qu'elle doit être plutôt comprise comme un être ouvert à la possibilité même de l'avenir dans lequel l'âme se rapporte à l'éternité comme à l'avenir. Dieu est l'avenir absolu à partir duquel le présent peut être autre chose que la distension de l'âme.

Kierkegaard souligne ainsi en quoi cette patience-espérance, qui se trouve au cœur de la temporalité chrétienne, n'est pas cette patience du concept envisagée par Hegel, car elle n'est ni le long travail propre au concept dans l'histoire, ni l'effort de totalisation et d'intériorisation du passé. En un sens, la patience comme attente de l'éternité libère d'un tel projet d'accomplissement de la subjectivité, dans la mesure où par elle l'homme renonce à l'idéal, issu de la peur du monde, d'une identité immuable et trouve sa stabilité, toujours transitoire, dans le bien de l'aimé, en échappant également à toute anesthésie de la résignation : l'endurance du monde devient par elle l'attente de l'inespéré. Or, selon la parabole du semeur, cette patience liée à la douleur et à l'humilité est agissante sans être un oubli de la finitude dans l'obstination utopique. Pour Kierkegaard, Dieu est la patience, en lui la patience est devenue une personne car il a tout supporté et tout donné, et c'est pourquoi l'homme en chemin vers Dieu avance dans la patience, vit dans la patience et aime la vérité dans la patience: il approche de Dieu en apprenant la patience, en s'y exerçant. Ainsi l'homme ne devient patient que par la patience de Dieu qui donne sens à la temporalité humaine en lui donnant un avenir parce qu'elle le fait entrer dans un rapport de personne à personne.

L'homme patient est alors celui qui reçoit le temps de Dieu, alors que l'impatient est celui qui échoue à se donner le temps et donc échoue à devenir une personne :

«Trop souvent, hélas! on prend l'impatience pour l'enthousiasme humble et obéissant; et l'impatient lui-même n'y est que trop enclin. Quand un homme est

32 Voir OC, 6, p. 202. 
accaparé du matin au soir par "la cause du Bien", dans le bruit, le vacarme et l'agitation incessante, quand il se démène dans le temps comme un malade sur son lit, rejette tous égards comme le malade ses vêtements, méprise le salaire du monde et se fait place parmi la foule, beaucoup pensent alors, et il s'imagine lui-même, qu'il est un inspiré. Cependant, il n'est rien moins que cela, puisqu'il est partagé et que le partage ne ressemble pas plus à l'enthousiasme que le tourbillon au vent soufflant toujours dans la même direction. Ainsi de toute impatience ; elle est une espèce d'emportement ; on le voit chez l'enfant qui ne veut pas se donner le temps, et chez cet homme partagé dont nous parlons qui ne peut supporter à la fois le temps et l'éternité. Il ne peut ni ne veut comprendre la lenteur du $\operatorname{Bien}(. ..) »^{33}$.

L'impatient donc ne se «donne pas le temps » au sens où il est incapable de cette synthèse du temps et de l'éternité, et dans sa peur du temps il vit dans l'instant vide de l'enthousiasme. Il n'accepte pas que le temps humain soit le temps de la patience et de l'attente du bien; il ne consent pas à se comprendre comme pauvre. Paradoxalement, dans cette temporalité inauthentique de celui qui ne comprend pas la lenteur du Bien, qui tel l'enfant veut tout immédiatement, l'horizon de l'éternité perd toute consistance et devient un rêve vaporeux que l'on tente d'atteindre par le saut impuissant de l'enthousiasme. Ceux qui sont «tout feu, tout flamme » ne vont jamais loin, parce qu'ils ne sont pas en chemin, mais en quelque sorte sautillent sur place. Vouloir toujours aller de l'avant sans comprendre que la lenteur est le chemin, c'est se condamner à vivre dans l'instant vide de la répétition. L'impatient va de l'avant en homme énergique, mais il risque alors de n'être qu'un histrion, un agité, qui n'est mis en mouvement que par lui-même et non par le Bien, et c'est pourquoi il va de l'avant sans avoir véritablement d'avenir. Dès lors, « se donner le temps » signifie en fait être ouvert, depuis sa finitude, à l'horizon du Bien qui me temporalise, et la patience se comprend donc comme le seuil de l'existence, comme ce qui permet d'entrer dans la contemplation et dans l'action. La patience n'est pas la résolution, mais ce qui la prépare et la rend possible ; elle est le temps de l'épreuve par lequel l'instant des retrouvailles avec le fils prodigue n'est pas vide ${ }^{34}$. De ce point de vue, Kierkegaard non seulement ne réduit pas la patience à la seule persévérance issue de la décision d'être maitre de soi, mais en outre il met en lumière l'unité des différentes dimensions de la patience : la capacité de supporter, d'attendre, d'être ouvert est aussi ce qui permet que la résolution ne soit pas vide, qu'elle ne soit pas un simple vouloir se vouloir, mais une vocation. Ainsi Kierkegaard revient sur la séparation moderne de la patience et de l'amour, qui a conduit à la

\footnotetext{
${ }_{33} \mathrm{OC}, 13$, Discours édifiants à divers points de vue, p. 62.

34 Sur cette épreuve avant l'épreuve, voir Marie-Christine Habbard, «Les malentendus de l'éthique », dans Soren Kierkegaard. Pensées et problèmes de l'éthique, Presses Universitaires du Septentrion, 2009.
} 
disparition de la patience et de l'amour en éthique, pour montrer que la passivité participe aussi de l'accomplissement de soi.

Il en va bien sûr ici d'une réflexion sur l'origine du sens, puisque le sens ne peut pas être reconduit à la seule activité d'une subjectivité, voire d'une intersubjectivité. Kierkegaard montre que le sens doit être compris sur l'horizon de l'éternité et donc qu'il engage la synthèse du temps et de l'éternité. Or l'éternité dont il s'agit dans cette élucidation de l'origine du sens n'est pas une éternité abstraite produit de l'imagination, mais l'éternité qui peut s'incarner dans le temps et constituer la plénitude de l'instant. La critique de l'impatience permet ainsi à Kierkegaard de ne pas s'en tenir à la seule volonté humaine pour revenir à l'attente du Bien source de toute compréhension. En conséquence, il n'y a pas de réflexion sur le sens qui ne se laisse questionner par le phénomène de l'impatience, dans lequel le temps disparait au profit d'un instant vide. D'une certaine façon, l'impatience est le corrélatif de l'ennui : l'impatient s'occupe pour surmonter sa peur du temps, son incapacité à faire face à la longueur du temps et également à la difficulté du choix. En effet, l'impatience est cette incapacité à laisser le temps passer, à respecter la durée propre des choses, des êtres, des communautés. Elle consiste finalement à forcer le monde à correspondre à ses intentions, à forcer autrui à s'adapter à ses projets, et également à se forcer soi-même, c'est-àdire à s'imposer un « soi » déterminé à l'avance, qui n'advient pas du temps lui-même. Dans la vie intellectuelle elle-même l'impatient peut être brillant, il peut multiplier les projets et les réalisations de façon indéfinie, tout en se révélant finalement stérile dans cette agitation issue de la vaine gloire que le monde d'aujourd'hui confond avec le sérieux de la réflexion. Pour expliquer cette temporalité du péché, la force des analyses de Kierkegaard est de ne pas expliquer l'impatience à partir d'une définition de l'homme, mais au contraire de rendre compte de l'existence humaine à partir de l'impatience. En effet, le paradoxe de l'impatience est qu'en elle l'homme cherche le bien, il veut le bien, mais sans être mis en mouvement par le Bien, sans être pris par son événement et sans vouloir endurer l'angoisse du dilemme propre au choix du choix. L'impatience n'est donc pas ici une qualité du sujet, mais elle se comprend plutôt comme une certaine façon d'être disposé à la vérité et au bien, et ainsi comme un mode de l'existence. Dans ce cadre, le propos de Kierkegaard n'est pas strictement ontique, mais il est également ontologique : l'impatience est effectivement ici une façon de ne pas être attentif, de ne pas être bienveillant, c'est-à-dire de ne pas veiller au Bien. Si toute vraie patience vient de Dieu, l'impatience est radicalement mienne, car elle demeure l'acte souverain d'un sujet qui veut posséder le monde et qui est incapable de vouloir que le monde advienne à luimême selon sa temporalité propre. En conséquence, ne pas se donner le temps est bien un mode de la temporalisation qui prive le soi et le monde de leur temps propre et donc qui les prive de leur être. Ainsi l'agitation de l'impatient le fait exister sur le mode de la fermeture et fait qu'il se 
temporalise «à vide », et c'est pourquoi l'impatient ne comprend pas : il ne parvient pas à accéder au sens de ce qu'il rencontre, il n'a pas d'expérience de la transcendance, car pour l'impatient rien n'existe pour soi, mais toujours seulement comme un moyen au service de sa gloire. L'homme sombre alors dans le désespoir lié à cette expérience de l'abîme entre le temps et l'éternité, entre l'existence présente et l'horizon vaporeux de l'imagination, tel celui qui veut être César ou rien. Un renversement total de son être se révèle alors nécessaire, afin de voir que la patience est la conscience que le bien nous oblige, qu'il nous lie à une tâche de compréhension et d'action.

Kierkegaard n'est pas le premier à décrire l'impatience comme la dispersion de l'homme dans le temps, ni à montrer que la patience est un rassemblement de soi dans le temps par la rencontre du Bien qui seule reconduit à soi, mais il insiste plus que d'autres sur le fait que l'homme patient, telle Anne, «a semé avec des larmes $»^{35}$ :

«Mais qu'est-ce alors que la patience ? N'est-elle pas le courage se chargeant librement de la souffrance inévitable ? L'inévitable c'est cela précisément qui veut briser le courage. Il y a chez l'homme de souffrance lui-même une résistance félonne alliée à la terreur de l'inévitable, et leurs forces unies veulent l'écraser ; malgré cela, la patience réside dans la souffrance, et ainsi justement elle se trouve libre dans la souffrance inévitable $»^{36}$.

On retrouve ici cette idée grecque que la patience est un courage : Ulysse dans l'Odyssée est celui qui a beaucoup supporté grâce à cette force d'âme accompagnée de raison, pour reprendre la définition du Lachès. Ce courage qu'il soit physique ou intellectuel est cette capacité à tenir bon grâce à la force d'âme et à l'intelligence de la situation. Ainsi, notamment face à la mort, l'homme est reconduit à ce qui peut constituer son humanité, à ce courage nécessaire à cette vie qui est un combat. En cela l'homme ne tient ferme dans le monde qu'en se tenant fortement à ce qui est stable, c'est-à-dire en se tournant vers les Idées. La patience provient alors à ce regard vers l'être qui permet de vivre sans se perdre au milieu de ce qui change sans cesse. L'objet de la patience est ici l'homme lui-même dans sa capacité à se conformer à une mesure qui est au-delà de lui et qui lui permet de faire face à la souffrance. L'existence humaine se trouve face à une seule alternative : ou la fermeté ou la mollesse. Kierkegaard reprend en grande partie une telle analyse, tout en ajoutant cependant que la résistance à l'inévitable qu'est la souffrance elle-même peut briser l'homme. Quoi qu'il en soit, la patience n'est pas une liberté en dépit de la souffrance ou qui proteste contre la souffrance, mais elle est une patience dans la souffrance. La perspective de Kierkegaard est donc

\footnotetext{
${ }^{35}$ Psaume, 126, 5 cité OC, 6, p. 206.

36 OC, 13, p. 115-116.
} 
bien ontologique : la patience est la modalité proprement humaine de l'être au monde qui fait que l'homme n'a ni le mode d'être de la chose, ni celui de l'animal. En effet, Kierkegaard ne parle pas seulement de l'excellence de la patience pour l'homme, au sens où elle serait utile à l'homme dans un monde de souffrances, mais il comprend la patience comme l'essence de la liberté humaine et donc comme la définition même de l'homme. Dans cette nouvelle considération de la patience, autrement dit non grecque, la patience ne dérive plus du courage, mais elle est plus originaire que lui :

«Aussi peut-on dire que la patience accomplit une merveille encore plus grande que ne le fait le courage, car celui-ci va librement à la souffrance qu'il pourrait éviter, tandis que la patience se rend libre dans la souffrance inévitable ; grâce à son courage, l'homme libre se laisse librement emprisonner ; mais grâce à sa patience, le prisonnier se rend libre - sans toutefois que le geôlier ait eu à ressentir peur et angoisse. L'impossibilité de se libérer extérieurement de la souffrance n'empêche pas la possibilité de s'en libérer intérieurement dans la souffrance, de s'en charger volontairement, lorsque par la patience on veut y consentir et accepter de s'y trouver $»^{37}$.

Cette libération intérieure de la souffrance est décisive, mais elle ne consiste justement pas à fuir la souffrance, à faire comme si elle n'était rien pour nous, puisqu'au contraire il s'agit bien de se charger volontairement de la souffrance, c'est-à-dire non pas faire comme si on voulait la souffrance, mais consentir à vivre avec cette souffrance dans une liberté finie. Cette attitude dans la souffrance n'est pas non plus la tentative désespérée de tenter de donner un sens à la souffrance en cherchant à quoi elle peut être utile, et elle doit plutôt être comprise comme l'acceptation libre de vivre avec cette souffrance. Autrement dit, la vrai patience ne cherche pas à relativiser la souffrance, ni à minimiser la dépossession propre à la souffrance, et c'est pourquoi elle est bien plus qu'un simple endurcissement, qui est une tentative vaine pour la surmonter en devenant moins sensible. Kierkegaard est très attentif à cette volonté trop humaine de fuir la souffrance en la relativisant, en minimisant sa réalité d'épreuve radicale, et c'est pour cette raison qu'il insiste tant sur le fait que la souffrance n'est pas rien, qu'elle n'est pas non plus la simple occasion de mettre à l'épreuve la volonté et ainsi de la purifier de tout reste de passivité. En effet, la patience n'est une libération intérieure que comme synthèse de la nécessité et de la liberté dans la résolution. Comme Kierkegaard l'écrit un peu plus loin dans le même texte, la patience est la souplesse de la réponse humaine à l'oppression de la nécessité : en elle l'homme plie sans rompre et ainsi dure dans la

\footnotetext{
37 OC, 13, p. 116.
} 
souffrance. Elle consiste à «faire de nécessité vertu», non au sens d'un renoncement, ni au sens d'une indifférence du sage qui sait conserver sa grandeur d'âme face aux plus grandes contraintes, mais par une temporalisation dans laquelle l'obligation de la nécessité ne supprime pas l'obligation du Bien. La plénitude du temps est le mouvement de consentir éternellement à vouloir tout souffrir, à vouloir ainsi dans le temps ce qui n'est pas de l'ordre du temps. De ce point de vue, l'homme patient est un «citoyen de l'éternité » et pour lui le temps n'est pas une simple dégradation de l'éternité. La liberté tient alors à la possibilité de vivre avec la souffrance parce que l'on est en souffrance du Bien. Tout cela conduit à reconnaittre que c'est à partir de l'amour de la vérité que le temps de la patience est un temps de l'accomplissement, car en lui l'homme, depuis sa souffrance, est tendu vers l'éternité. L'homme patient n'est pas celui qui fuit la souffrance ou celui qui veut le Bien malgré la souffrance, mais il est celui qui demeure « avec le Bien dans la décision, en voulant tout souffrir, en voulant la souffrance imposée par la nécessité $»^{38}$. Dans ces considérations totalement étrangères au dolorisme, Kierkegaard met en évidence que la patience est le triomphe de l'homme de bien en cette vie :

«Si l'on cherchait le terme adéquat exprimant la constante victoire du bien, lequel conviendrait mieux que celui-ci : le franc-courage est capable, au sein de la souffrance, de retirer au monde sa puissance $»^{39}$.

Selon Kierkegaard, une telle possibilité vient de Dieu, c'est Dieu qui donne la patience, mais elle est également pour Dieu, elle est la capacité de pâtir pour Dieu dans le témoignage. Loin d'être une capacité a priori d'un sujet isolé, la patience est une ouverture et non un endurcissement, elle est l'acte de se communiquer soi-même dans le temps, c'est-à-dire un don de soi comme respect de ce vers quoi on se tourne. Ainsi comprise, la patience ne peut être considérée comme une qualité naturelle que l'homme posséderait à l'avance, mais elle provient de la rencontre elle-même ; plus précisément, elle est à la fois une autodétermination et ce qui est reçu dans l'épreuve, et c'est pourquoi elle est un paradoxe. Il est alors possible de dire que c'est la patience qui fait l'homme et non l'homme qui produit la patience, puisque l'objet de la patience, le Bien, déborde l'homme patient et excède ses forces. Dès lors vouloir l'éternel dans le temporel cela revient à s'élever à une existence à l'impossible qui fait de l'homme un être spirituel se laissant reconduire à lui-même non par une chose, ni par soi, mais par l'événement du Bien. Cela conduit à reconnaitre que la vraie patience ne porte pas sur soi et que ce n'est jamais soi que l'on attend, puisque pour attendre il faut être pris par autre chose que soi, par un sens qui ne relève pas de notre pouvoir de constitution en

\footnotetext{
38 OC, 13, p. 117.

39 OC, 13, p. 324.
} 
objet. Ainsi la patience est le style d'une existence essentiellement transitive : l'homme patient est celui qui vit dans l'attente de l'éternité, sans prétendre la contenir. La patience n'est donc ni l'absence de volonté dans la soumission à un destin, ni une volonté pure que l'on se donnerait soimême, mais elle doit se comprendre comme une volonté brisée par le Bien ; c'est en ce sens que seul un homme de volonté peut devenir patient ${ }^{40}$.

Bien sûr, toute attente n'est pas portée par l'bypomoné et peut n'être que l'attente sans espérance de ce qui ne se produit pas et Beckett dans En attendant Godot a pu décrire l'enfer de cette temporalité vide, sans fin ni commencement, dans laquelle l'événement est impossible, comme est impossible la moindre résolution et la plus simple action. Il est même possible de se demander si un tel vide n'est pas la caractéristique fondamentale de l'enfer, dans la mesure où dans ce vide du désespoir, de l'ennui ou de la folie s'accomplit une impassibilité radicale qui met littéralement l'individu hors d'atteinte de tout événement et l'enferme en lui-même. Dans cette temporalité fermée à tout avenir et totalement close rien ne se passe, aucune vraie parole n'est possible, car l'homme fait face à son néant sans parvenir à redevenir un visage éclairant, une personne. Le seul échappement envisageable de cette existence résignée à l'inertie tient à la capacité de retrouver la source de la vraie patience, qui est l'amour en ce qu'il est l'esprit lui-même en acte, et non une simple qualité qui pourrait s'ajouter à l'esprit, comme a pu le montrer saint Augustin. Par rapport à toutes les figures d'une temporalité bloquée dans un sens figé, y compris dans une attente figée, l'amour vient briser ce cercle de l'immanence, vient libérer de la stabilité factice de l'endurance vicieuse, pour rendre l'homme à la véritable source du sens. Dès lors, l'amour compris comme charité vient briser également le partage du monde par les catégories issues de la convoitise ; ce sont notamment les catégories fondamentales de la temporalisation de soi qui se trouvent bouleversées. En effet, la vraie patience ne saurait être une hâte et dans sa résistance à l'impatience elle fait de la lenteur une vertu du cheminement, cependant cette lenteur qui sait attendre que chaque fruit vienne à maturité n'est pas non plus une apathie, et elle n'est pas incompatible avec l'évidence d'une urgence, avec la conscience impérative d'une tâche à accomplir en propre. La vraie lenteur ne lambine pas, elle n'est pas ce désœuvrement de l'ennui dans lequel on vit pour soi, même si c'est un soi vide, et elle est au contraire une tension extrême qui ne vient pas de soi, de la visée d'une Idée téléologique, mais de l'ouverture même à l'altérité comme lors de la prière. Dans l'amour, l'action conserve la sérénité de l'espérance sans se laisser pour autant différer, parce qu'elle est un engagement de son être tout entier, une œuvre. Ce que nous avons à faire, nous devons le

\footnotetext{
40 Sur la « volonté brisée », voir Journal, tome 5, op. cit., p. 363 « Seul un homme de volonté peut devenir chrétien. Il est le seul en effet à en avoir une qui puisse être brisée. Mais un homme de volonté dont la volonté est brisée par l'absolu ou par Dieu, c'est un chrétien. Plus la volonté a de force naturelle, plus profonde peut devenir la brisure et mieux on est chrétien ».
} 
faire «vite », car c'est à nous de le faire, c'est une tâche reçue en propre, et nous ne devons pas nous en défaire dans le vagabondage ou dans la précipitation. Il y a ainsi une synthèse de la lenteur et de l'urgence dans la vraie patience, parce qu'elle n'est pas comme dans l'endurance vicieuse un non à tout ce qui menace mon autonomie, mais un oui qui est l'amour même. De ce point de vue, la figure de Job met en évidence que la patience ne consiste pas à se préserver en attendant que l'orage passe. Dans l'épreuve Job perd tout jusqu'à « la force et la raison de vivre ${ }^{41}$ », cependant sa patience montre que ce n'est pas la souffrance qui le sauve, mais l'amour, et c'est pourquoi «Job est béni et il a tout reçu au double. - Cela s'appelle une reprise ${ }^{42} 》$ : celui qui a tout perdu reçoit tout au double dans la patience de l'amour, y compris lui-même. La patience est ici une vrai patience, c'est-à-dire une véritable pâtir avec sa dimension de perte, mais celui qui a tout perdu reçoit la grâce de tout pouvoir recevoir. L’épreuve est alors dépassée, au sens hégélien de l'Aufhebung, dans le oui.

Bien évidemment, Kierkegaard élucide lui aussi le lien eidétique de l'hypomoné biblique et de l'amour, en montrant que la patience est la temporalisation même de l'amour, qui est la synthèse de l'éternel et du temporel. Pour comprendre cela il est impératif de dépasser une conception purement humaine de l'amour, qui fait de la réciprocité une condition. La vraie patience n'est pas comparative, elle n'est pas l'équilibre des efforts réciproques, car une telle patience conditionnée demeure limitée, elle ne peut pas tout. Kierkegaard cite saint Paul «L'amour est patient » (1 Cor. 13), et c'est dans cette patience propre à l'amour qu'il peut supporter l'incompréhension, l'ingratitude, la colère d'autrui ${ }^{43}$. A l'impossible nul n'est tenu pourrait-on répondre avec Aristote, mais justement «tout supporter » n'est pas ici un projet que l'homme se donne dans une volonté herculéenne de surmonter toute finitude, ce n'est pas non plus le projet absurde de tout accepter dans une résignation sans fin. Pour élucider le sens de cette folie à l'égard du monde comme de la philosophie, Kierkegaard veut mettre en lumière que l'amour qui supporte tout, telle la mère qui supporte les bêtises de ses enfants, signifie la vérité sociale de l'existence : «L'amour n'est pas une qualité donnée pour elle-même, mais bien une qualité par laquelle ou dans laquelle tu es pour d'autres $»^{44}$. Ainsi la conversion de l'endurance vicieuse en une vraie patience, qui est endurance de l'altérité par la grâce divine, réside dans ce passage au «pour autrui » de l'existence. Dans ce renversement de la perspective, la patience n'est pas cette simple force propre qui permet de supporter autrui et de faire de l'endurance de son altérité une simple cause occasionnelle de l'intelligence du sens de son devoir. Il faut même reconnaître que d'une certaine façon l'autre est

\footnotetext{
41 La reprise, trad. Nelly Viallaneix, Paris, Flammarion, 1990, p. 143.

42 Ibid. p. 156.

43 Les cuvres de l'amour, OC 14, p. 203.

44 OC, 14, p. 206.
} 
« insupportable » et que seule la foi permet de l'attendre, de l'endurer. En retour, la patience d'autrui est ce qui reconduit à lui-même et ce qui donne à ce moi de s'accomplir. Le chemin de moi à moimême passe par la patience d'autrui sans laquelle je demeure enfermé en moi. En conséquence, le sens de ce que je dois faire pour autrui ne peut pas venir de moi seul, et c'est pourquoi dans cette « seconde éthique » qui élucide la patience chrétienne, la patience supporte tout parce qu'elle est agissante, parce qu'elle agit pour et à partir d'autrui. De ce fait, la patience est le chemin, car il s'agit d'un chemin qui s'ouvre à partir du prochain lui-même. On peut alors comprendre que si la patience supporte tout, ce n'est pas parce qu'elle serait héroïque, mais parce qu'elle est l'expression de l'amour, parce qu'en elle on existe pour l'autre dans l'humilité et l'espérance. Une telle patience de l'amour ne repose pas sur l'évidence théorétique de ce qui adviendra, mais sur l'évidence pratique que c'est à moi d'attendre et d'espérer, même en dehors de toute raison objective. Or cette patience dans l'espérance fait de l'autre mon fondement, non au sens d'une Grundlegung, mais d'une origine qui me temporalise. La patience devient alors une intelligence du sens dans la mesure où elle laisse le prochain se signifier lui-même. Il est alors possible de dire que la seule patience véritable vient de la charité, car elle est la grâce d'être patient pour autre chose que soi, et c'est pourquoi non seulement elle supporte tout, mais elle est également ce qui rend possible une véritable unité des hommes. Le sens est éthique comme dira plus tard Levinas, il ne relève pas d'abord d'une métaphysique de la subjectivité close ou d'une métaphysique de l’intersubjectivité. En conséquence, la patience ne saurait être une obligation fondée sur l'universalité de la loi morale, et l'amour justement, qui n'est pas une valeur, donne à comprendre que la patience est une obligation plus originaire que celle fondée sur des valeurs : on donne sa vie pour le prochain et pas au nom d'une valeur, et la seule véritable obligation a lieu dans une relation de personne à personne. Ainsi la vérité qui m'appelle n'est jamais impersonnelle et abstraite, mais elle s'incarne toujours dans un visage qui se tourne vers moi. Comme le souligne Kierkegaard ${ }^{45}$, il y a dans tout amour véritable une pudeur sacrée, qui signifie que l'homme a conscience de son insignifiance devant la réalité de ce qu'il aime, à savoir le Bien, qui est pour lui une personne. Il faut en conclure que dans la patience le sens est l'excès de ce qui s'annonce à moi au-delà du possible et du pensable, et c'est pourquoi l'amour ne se voit pas, il ne se reconnait pas directement dans une manifestation, mais il exige qu'on y croit, car le sens qui se donne en lui dépasse toute représentation. Ainsi la patience ne suppose pas un esprit pur de toute passivité, mais un cœur pur de toute condition, mais, Kierkegaard insiste, un cœur pur est un cœur lié à Dieu dans une obligation qui ne prend jamais fin ${ }^{46}$.

\footnotetext{
45 OC, 14, p. 316 : «Nous avons ici la pudeur sacrée inséparable de tout amour véritable. Car la pudeur de la femme, au-dessus des choses de ce monde, permet à celle-ci de se sentir supérieur à ce qui la choque et la blesse ; mais la pudeur sacrée est le sentiment qu'à l'homme de son insignifiance devant la réalité de Dieu ».

46 OC, 14 , p. 136.
} 
L'opposition constante entre l'endurance vicieuse et l'endurance de l'altérité a montré que la patience est le véritable lieu de partage entre deux styles d'existence, mais également entre deux types de philosophie : la philosophie impatiente, même dans la patience du négatif qui est pure spéculation, et la philosophie patiente, qui demeure en dette éternelle vis-à-vis de la manifestation. La mise en évidence de ce lien d'essence entre patience et amour donne donc à comprendre que le sens n'est pas réductible à ce qui peut être obtenu par un processus toujours plus poussé d'idéalisation, fut-elle intersubjective, et Kierkegaard ouvre à une nouvelle intelligibilité du sens en décrivant la transitivité de l'existence à travers la patience, puisqu'il s'agit de suspendre le souci de comprendre le monde à partir de la seule élucidation de soi : le sens est ce qui me prend et m’oblige avant tout voir. En cela, le sens de mon devoir ne peut pas venir de la simple universalisation de ma maxime subjective et d'une pure transparence à soi dans le respect pour la loi morale ${ }^{47}$. Depuis mon opacité mon devoir se comprend dans l'amour, c'est-à-dire depuis cette patience qu'autrui me donne en se laissant aimer. L'amour est connaissance, non au sens où il me rendrait transparent à moi-même, mais parce qu'aimer autrui, c'est toujours saisir le sens de sa mission pour lui. De cette façon, c'est l'amour reçu qui en moi prend patience et rend possible la résolution éthique. Autrement dit, la volonté brisée de la patience donne à comprendre le sens de l'altérité en mettant en lumière l'altérité du sens, car elle accueille tout être comme une promesse de sens.

\footnotetext{
${ }^{47}$ Sur amour et respect voir notre ouvrage, L'intelligence de la pitié, Paris, Cerf, coll. La nuit surveillée, 2003.
} 